\title{
RECIENTES EXPERIENCIAS Y TRES CASOS SIGNIFICATIVOS DE INSPECCIÓN, DIAGNÓSTICO Y REPARACIÓN DE DAÑOS DE VIGUETAS PREFABRICADAS CON CEMENTO ALUMINOSO
}

\author{
(RECENT EXPERIENCES AND THREE SIGNIFICANT CASES OF INSPECTION, DIAGNOSTIC \\ AND REPAIR OF DAMAGE IN PRECAST BEAMS WITH HIGH ALUMINA CEMENT)
}

\author{
A. Delibes, J. Díaz Lozano, E. González Valle, J. Ley y P. López Sánchez \\ INTEMAC / MADRID / ESPANA
}

Fecha de recepción: 11-XII-92

$615-9$

\begin{abstract}
RESUMEN
El hormigón de cemento aluminoso se utilizó en España entre los años 50 y 70 para la producción de viguetas de forjado de hormigón pretensado, empleando el cemento, fabricado por Cementos Molins, desde 1928, bajo la licencia francesa de cementos Lafarge. Los daños y hundimientos ocurridos recientemente han conducido a nuevas investigaciones en edificios sospechosos, que han llegado a estimarse en más de 100.000 viviendas en Barcelona y de 50.000 en Madrid, cifras que nos parecen exageradas. Este trabajo expone las experiencias de INTEMAC en los dos últimos años, así como su metodología para la inspección, toma de muestras y ensayos que permiten establecer el correspondiente diagnóstico y la propuesta de refuerzo en su caso.
\end{abstract}

SUMMARY

High Alumina Cement (HAC) concrete was used in Spain from 1950 to 1970 in the production of prefabricated prestressed joist for floor slab construction, using cement produced by Cementos Molins from 1928 under licence from Lafarge (France). Recent collapses have led to new investigations on buildings under suspiction of having HAC concrete joists. The number of dwelling under suspiction has been evaluated elsewhere in 100,000 in Barcelona and 50,000 in Madrid, these ciphers are, in the authors' opinion, exagerated. This paper explains INTEMAC's experiences and methodology for the in-situ inspection, sampling and testing, leading to the corresponding diagnosis and, in some cases a proposal for repair and maintenance practices.
1. EL USO DEL CEMENTO ALUMINOSO PARA LA FABRICACIÓN DE VIGUETAS EN ESPAÑA

En 1824 J. Aspdin patenta en el Reino Unido el cemento portland cuya utilización se extiende a lo largo del siglo pasado, en cuya segunda mitad tiene lugar el desarrollo de elementos de hormigón armado cuyo conocimiento estructural no tiene lugar hasta principios del siglo actual.
En 1908 J. Bied patenta en Francia el cemento aluminoso, en que se sustituyen los silicatos cálcicos por aluminatos cálcicos, subrayándose que de este modo se obtiene un hormigón de alta resistencia a los sulfatos y por tanto a los terrenos yesíferos. El paréntesis de la Primera Guerra Mundial hace que esta patente no se desarrolle industrialmente hasta 1920 en que comienza la fabricación en Francia por Cements Lafarge. La patente Lafarge se desarrolla rápidamente en todo el mundo: 


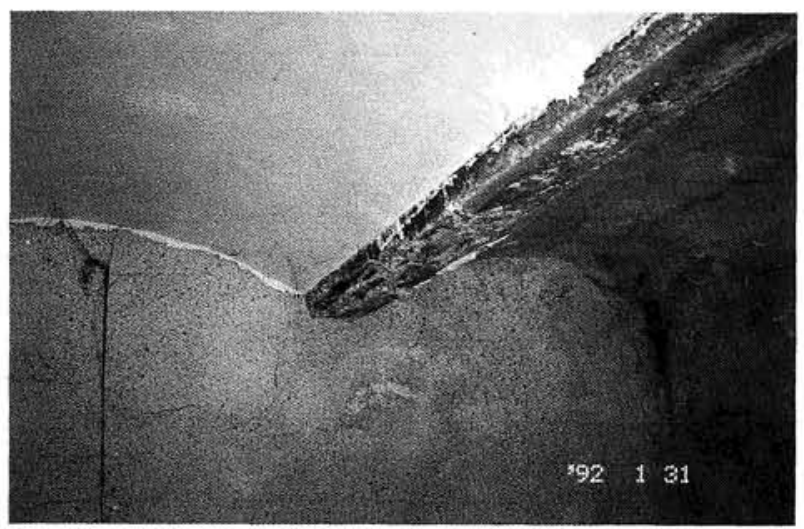

Suela inferior de una vigueta fabricada con cemento aluminoso en que se observan daños graves por corrosión de las armaduras.

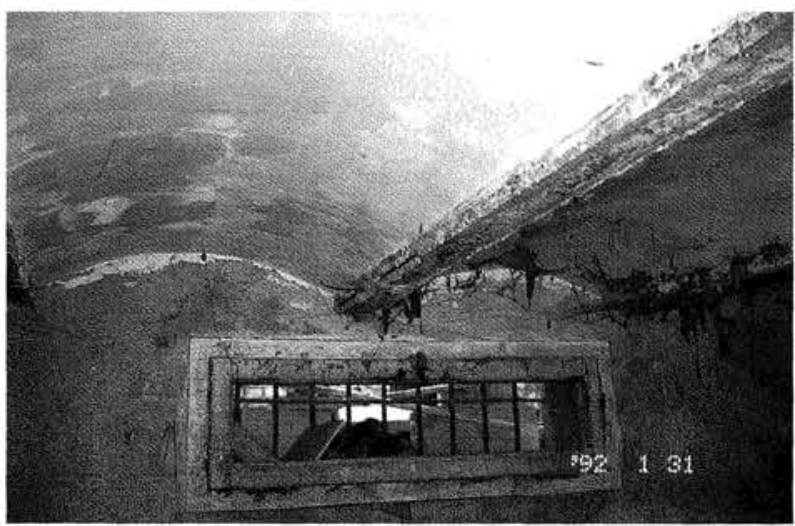

Daños en la zona de entrega del apoyo de una vigueta prefabricada con hormigón de cemento aluminoso.

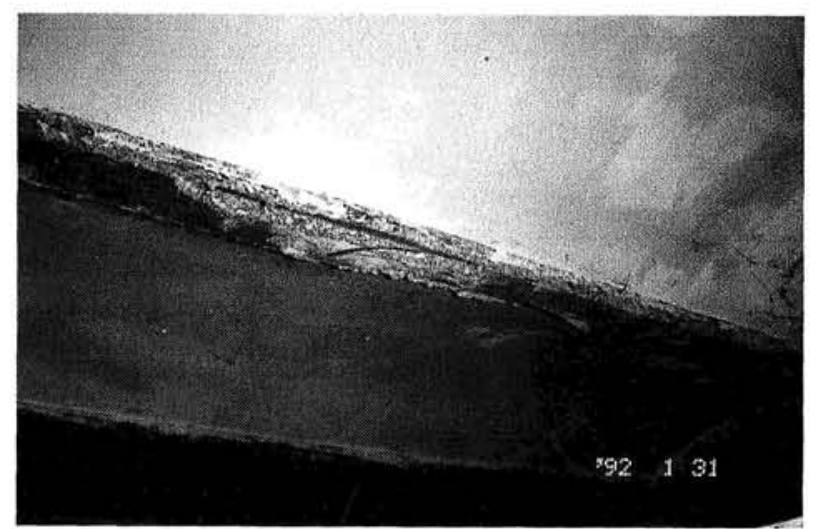

Daños en centro de vano con rotura de armaduras pretensadas. (C) Consejo Superior de Investigaciones Científicas Licencia Creative Commons 3.0 España (by-nc)
En 1924 comienza la fabricación en USA, en 1925 la fabricación bajo patente en el Reino Unido y en 1928 la fabricación en España bajo patente francesa.

Entre 1950 y 1970 tiene lugar en España un auge espectacular de la construcción donde se desarrollan y usan con profusión forjados de hormigón pretensado de viguetas o semiviguetas prefabricadas. Durante este período se produce una media de 2.192 t/año de cemento aluminoso, lo que representa una fracción de $1 / 1100$ de la producción de cemento portland en nuestro país. Paralelamente tiene lugar un desarrollo análogo en algunos países europeos como por ejemplo en Inglaterra donde la producción de cemento aluminoso alcanza fracciones de la producción total de cemento muy superiores. La producción, durante 20 años, supone la puesta en obra de 44.000 toneladas de cemento aluminoso, equivalentes a unos $120.000 \mathrm{~m}^{3}$ de hormigón de cemento aluminoso. Si toda la producción de cemento se hubiese empleado en la prefabricación de viguetas, el volumen de hormigón indicado correspondería aproximadamente a $2.500 .000 \mathrm{~m}^{2}$ de forjado de viguetas o semiviguetas prefabricadas, es decir a unas 250.000 viviendas de $100 \mathrm{~m}^{2}$. Esta cifra es ligeramente inferior pero muy aproximada a las estimaciones realizadas por el Ministerio de Obras Públicas y Transportes que se citan más adelante.

En general, la prefabricación de viguetas se realiza en esos años con una tecnología y calidad bastante deficientes, lo que no impide que fabricantes "serios" utilicen el mismo procedimiento.

El gran éxito de la aplicación del cemento aluminoso a la prefabricación de viguetas es debido a su alta resistencia inicial, en contraste con su propiedad de resistencia a los sulfatos en que se basaba la patente de J. Bied. Otra aplicación es el uso en construcciones refractarias. En las evaluaciones anteriores hemos supuesto que, al menos en España, la propiedad de alta resistencia inicial fue determinante de un uso casi exclusivo en la prefabricación de viguetas.

A partir de los años $60 \mathrm{y}$ primeros de los 70 se detectan y estudian distintos casos de daños por degradación de cemento aluminoso y corrosión de las armaduras pretensadas. Así, en 1962 comienza la prohibición del uso 
de cemento aluminoso en aplicaciones estructurales en varios "länder" alemanes. En 1970 se establecen limitaciones de uso en Francia, donde se había extendido muy poco el empleo de cemento aluminoso para la prefabricación de viguetas. En 1973 se prohibe prácticamente la utilización de cemento aluminoso en España para aplicaciones estructurales.

En Inglaterra tiene lugar una gran polémica sobre la limitación o prohibición del uso de cemento aluminoso. Tras los accidentes de las cubiertas de los colegios Stepney y Camden, así como el de la Universidad de Leicester en el Reino Unido, la polémica lleva finalmente a la prohibición del uso estructural del cemento aluminoso.

La prohibición en España se debe a la influencia de los accidentes estudiados en otros países europeos, así como algunos casos aislados detectados en zonas costeras y forjados de cubiertas. Estos casos continuan detectándose durante las dos décadas que van de 1970 a 1990. En 1991 tiene lugar la gran alarma de los hundimientos de Barcelona que se hace extensiva a otras ciudades como Madrid, donde el diario El País de 7 de junio de 1992 estimaba en 50.000 a 110.000 viviendas afectadas por patología del cemento aluminoso. En 1990 el Ministerio de Obras Públicas y Transportes firma un acuerdo con la Comunidad Autónoma Canaria para el establecimiento de ayudas para la reparación de daños producidos en las zonas afectadas. A partir de la alarma catalana, el Consejo de Ministros acuerda en sesión de 12 de julio de 1991 la creación del fondo estatal de ayudas, por el que el MOPT aportará 20.000 millones de pesetas en ayudas a las comunidades autónomas, que deberían aportar cantidades análogas. Las comunidades beneficiadas son Canarias, Cataluña (con el $60 \%$, es decir 12.000 millones), Baleares, Murcia y Valencia. Las estimaciones del MOPT llevan a considerar que existen en España 300.000 viviendas en que se emplea hormigón de cemento aluminoso, cifra bastante próxima a las 250.000 que hemos estimado corresponden a la producción entera de cemento aluminoso entre 1950 y 1970.

Hasta la fecha no se ha puesto en marcha un sistema global de inspección, evaluación y reparación de las construcciones afectadas.

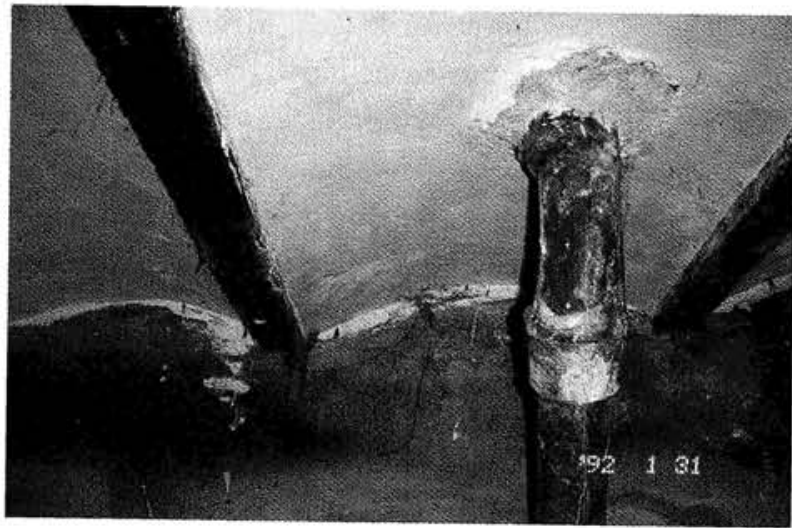

Fisuración longitudinal en zona de apoyo con aporte de humedad por la cercanía de una bajante.

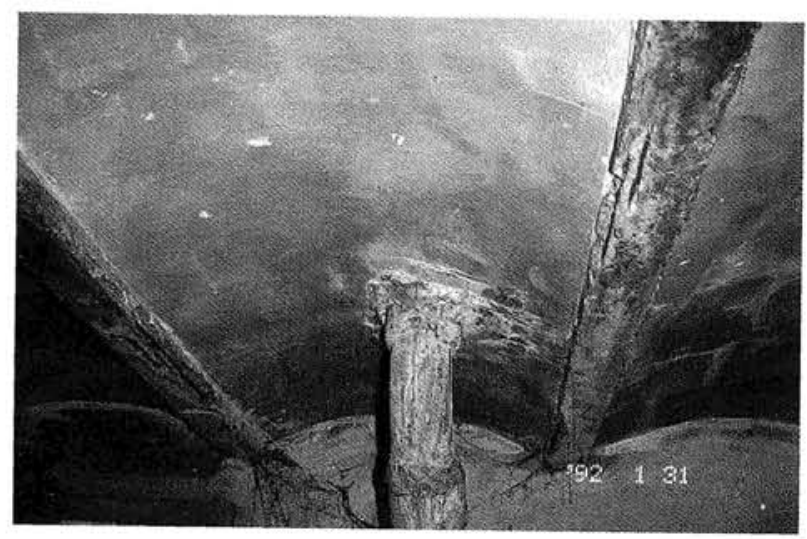

Descantillado del hormigón por corrosión de armaduras en zona húmeda, próxima a la bajante.

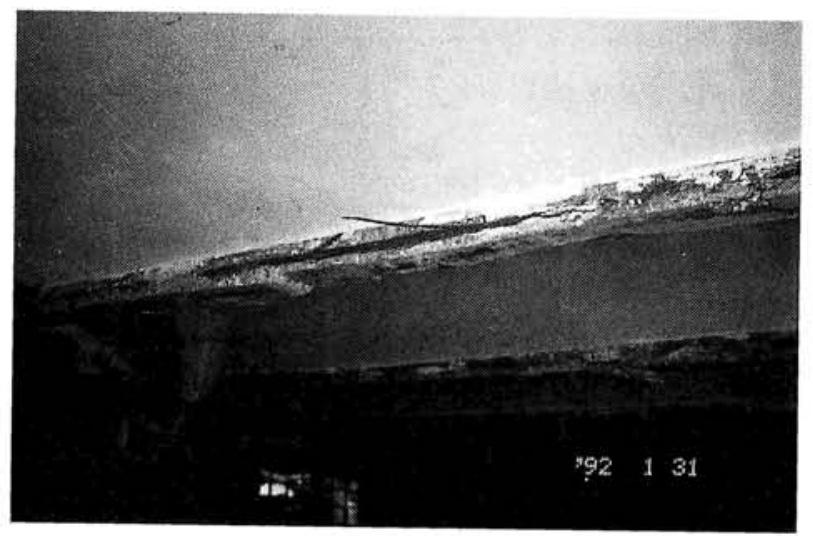

Corrosión y rotura de armaduras con desprendimientos del recubrimiento de hormigón en zona próxima a una bajante. 


\section{LA DEGRADACIÓN DEL HORMIGÓN DE CEMENTO ALUMINOSO}

La degradación del hormigón de cemento aluminoso tiene su raíz en el proceso mismo de hidratación de este tipo de cemento. El proceso de hidratación puede dar lugar a dos sistemas de cristales de aluminatos cálcicos: cristalización en el sistema hexagonal, metaestable, de compuestos de aluminatos monocálcico y bicálcico con 8 a 10 moléculas de agua, o bien cristalización en el sistema cúbico, estable, de aluminatos tricálcicos con 6 moléculas de agua acompañados de aluminatos (gibsita) estables. El primer proceso de hidratación tiene lugar con temperaturas inferiores a $25^{\circ} \mathrm{C}$. El segundo proceso tiene lugar con temperaturas de $40^{\circ} \mathrm{C}$. En condiciones de temperatura normales el cemento cristaliza en el sistema hexagonal sufriendo con el tiempo un proceso de conversión al sistema cúbico, con pérdidas de resistencia y aumento muy notable de la porosidad. Esta conversión se ve favorecida por las condiciones de alta temperatura y humedad.

Así, en el informe realizado por el Building Research Establishment (BRE) en 1988 se indica que la resistencia del hormigón tras la conversión puede pasar de un valor medio de $500 \mathrm{~kg} / \mathrm{cm}^{2}$ a $260 \mathrm{~kg} / \mathrm{cm}^{2}$ y el valor característico verse aún más reducido como consecuencia del aumento de la dispersión en el valor de la resistencia: pasando de unos $400 \mathrm{~kg} / \mathrm{cm}^{2}$ a $170 \mathrm{~kg} / \mathrm{cm}^{2}$. En ambientes húmedos la reducción de resistencia puede ser un $30 \%$ mayor.

Independientemente de los procesos de conversión cristalina, los aluminatos cálcicos del cemento aluminoso pueden verse afectados por carbonatación mediante mecanismos diferentes a los producidos en el cemento portland. Estos procesos pueden afectar tanto a las fases hexagonales como a las fases cúbicas, disminuyendo la reserva alcalina del hormigón desde valores cercanos a $\mathrm{pH}=11$ hasta $\mathrm{pH}=6-7$.

Un caso particular de las transformaciones por carbonatación es la hidrólisis alcalina, que produce, en presencia de álcalis y $\mathrm{CO}_{2}$ o carbonatos alcalinos, una disminución sensible de resistencias mecánicas y basicidad, con un incremento de la porosidad.

En todos los casos el resultado es una rápida corrosión de las armaduras que ha llevado, en la mayor parte de los accidentes investigados, al hundimiento de los forjaodeos afejectadosipiror de Investigaciones Científicas

\section{INVESTIGACIÓN DE LOS DAÑOS}

\subsection{Inspección ocular}

El estudio e investigación de los daños debe comenzar siempre por una inspección ocular bastante detallada que permita establecer la tipología de los forjados, su geometría, existencia de zonas con aportaciones exteriores de humedad (filtraciones, condensaciones, proximidad a bajantes), contactos del presunto hormigón de cemento aluminoso con hormigón de cemento portland (existente siempre en las zonas de apoyo con entrega a vigas hormigonadas in situ o correas de atado), posible degradación del hormigón, existencia de fisuras paralelas a las armaduras, existencia de manchas de corrosión, existencia de armaduras descubiertas, grado de oxidación de las armaduras, etc.

En muchos casos reales la inspección ocular ha permitido descartar la existencia de hormigones de cemento aluminoso dada la tipología de las viguetas de forjado o por tratarse de forjados hormigonados in situ. También es conocido el caso de un error cometido en la investigación de un edificio en Madrid donde se dictaminó la existencia de cemento aluminoso a partir de ensayos sobre muestras de hormigón procedentes de pilares vertidos in situ. La simple observación de la procedencia de las muestras en que se basó el dictamen hubiera bastado para descartar prácticamente la existencia de cemento aluminoso en este tipo de elementos.

Por otro lado, la inspección ocular permite una primera evaluación del nivel de daños y de degradación existentes, permite clasificar la estructura en zonas con diferente nivel de riesgo o bien en zonas húmedas y zonas secas y establecer, en consecuencia, un plan de toma de muestras eficaz.

\subsection{Toma de muestras}

Si se programa la realización de ensayos mecánicos es necesario proceder a la toma de muestras por métodos destructivos. Estos ensayos requieren cortar secciones enteras de viguetas o semiviguetas y en muchos casos puede ser más conveniente obtener muestras constituidas por piezas completas que permitan realizar ensayos a flexión en laboratorio o bien tallar muestras para la realización de ensayos a compresión y para la observación de las armetpdintras mesdelaconstruccion.revistas.csic.es 


\subsection{Ensayos físicos y mecánicos}

El ensayo físico más común es la determinación de la porosidad del hormigón en laboratorio. Permite estimar el nivel de degradación del hormigón y por tanto evaluar el grado de conversión del hormigón de cemento aluminoso. La toma de muestras correspondiente es un proceso semidestructivo que no requiere la rotura completa de la sección de la vigueta o semivigueta.

Con la obtención destructiva de muestras que conserven la sección completa pueden realizarse, tras el debido tallado con sierra de disco de diamante de porciones de semiviguetas adecuadas, ensayos mecánicos de resistencia a compresión o tracción del hormigón y también de las armaduras. Sobre las mismas muestras puede medirse la geometría real de las piezas afectadas y la cuantía y disposición de las armaduras, estableciendo desde el primer momento si se trata de piezas simplemente armadas o piezas pretensadas. Los ensayos mecánicos del hormigón permiten estimar el grado de degradación del material o el nivel de conversión del hormigón de cemento aluminoso, y son también la base para la realización de un análisis de la seguridad estructural que permitirá juzgar el grado de urgencia de la reparación o resfuerzo.

\subsection{Análisis químico}

Sobre las muestras anteriores pueden realizarse ensayos químicos o más propiamente físico-químicos que permitan diagnosticar la existencia de cemento aluminoso, el grado de conversión del cemento aluminoso, el nivel de carbonatación del hormigón y la futura evolución de los daños. Los ensayos disponibles para alcanzar dichos objetivos son el análisis mineralógico de los áridos, el análisis químico convencional de la fracción fina del hormigón, la difracción por rayos $\mathrm{X}$ y el análisis termogravimétrico. Requieren generalmente la obtención de muestras de tamaño tal que pueden considerarse como pruebas destructivas, pues en muchos casos reales es necesario obtener muestras que contengan la sección completa de la vigueta o semivigueta.

En los ejemplos que exponemos más adelante se dan algunos detalles sobre la metodología de ensayo aplicable y los procesos de interpretación de los resultados.

\subsection{Análisis de seguridad y propuesta de refuerzo}

Con los datos obtenidos durante la inspección ocular y el proceso de toma de muestras y ensayos es necesario siempre realizar un análisis de la seguridad estructural de los elementos afectados. Dicho análisis permitirá dictaminar sobre el nivel de seguridad actual de los forjados y establecer prioridades para su reparación o refuerzo. Asimismo permitirá estudiar los sistemas de reparación que se juzguen más adecuados. En general la intervención de INTEMAC en los casos reales que describimos a continuación puede verse limitada a una primera fase de inspección y ensayos que excluya el análisis de seguridad y sobre todo el estudio y propuesta de soluciones de reparación que generalmente requieren un grado de investigación bastante completo y detallado que, por razones obvias de economía, debe constituir una segunda fase de la investigación.

\section{CASOS REALES INVESTIGADOS POR INTEMAC}

Desde 1970 a 1990 INTEMAC ha participado en diversas investigaciones sobre casos detectados en zonas costeras, principalmente, de la geografía española. A partir de 1991 en que se produce la gran alarma ocasionada por los accidentes del Turó de la Peira de Barcelona, este Instituto ha realizado investigaciones o participado en las dirigidas por otras organizaciones en multitud de casos. Hemos seleccionados tres de ellos como los más significativos e interesantes entre los que implican un trabajo de investigación bastante completo y detallado, descartando los asuntos en que INTEMAC ha participado, en general a través de sus servicios de laboratorio, con otras organizaciones. Estos estudios detallados constituyen una experiencia bastante amplia constituida por 13 expedientes distintos, cuyo alcance se resume en el Cuadro n. ${ }^{0}$ l. En total figuran unos $200.000 \mathrm{~m}^{2}$ de forjado que, en algunos casos, ha resultado construido con hormigón de cemento portland, lo que ha podido dictaminarse tras la realización de los análisis químicos oportunos.

En lo que sigue se describen con mayor detalle las investigaciones realizadas en los casos numerados 1,2 y 8 que se han seleccionado como representativos de la metodología aplicada por INTEMAC.

http://informesdelaconstruccion.revistas.csic.es 


\section{CUADRO No}

ASUNTOS CON DAÑOS POR DEGRADACIÓN DE CEMENTO ALUMINOSO ESTUDIADOS POR EL DEPARTAMENTO DE PATOLOGÍA DE INTEMAC EN EL PERÍODO 1991-1992

\begin{tabular}{|c|c|c|c|c|c|c|}
\hline $\begin{array}{l}\text { Número } \\
\text { de Orden }\end{array}$ & $\begin{array}{l}\text { Tipo de } \\
\text { Edificio }\end{array}$ & SITUACIÓN & FECHA & PROPIEDAD & SUPERFICIE & COMENTARIOS \\
\hline 1 & $\begin{array}{l}\text { Viviendas y } \\
\text { Locales }\end{array}$ & Cataluña & 1991 & $\begin{array}{l}\text { Organismo } \\
\text { Público }\end{array}$ & $\begin{array}{l}192 \text { viviendas } \\
32 \text { Locales } \\
\left(22.400 \mathrm{~m}^{2}\right)\end{array}$ & $\begin{array}{l}\text { Estudio completo y } \\
\text { propuesta de refuerzo }\end{array}$ \\
\hline 2 & Hospital & Levante & 1992 & Administración & $30.000 \mathrm{~m}^{2}$ & $\begin{array}{l}\text { Estudio completo y } \\
\text { propuesta de refuerzo }\end{array}$ \\
\hline 3 & Hospital & Levante & 1992 & Administración & $21.000 \mathrm{~m}^{2}$ & $\begin{array}{l}\text { Estudio completo y } \\
\text { propuesta de refuerzo }\end{array}$ \\
\hline 4 & Hospital & Levante & 1992 & Administración & $3.000 \mathrm{~m}^{2}$ & Inspección ocular \\
\hline 5 & Viviendas & Cataluña & 1992 & Privada & $1.350 \mathrm{~m}^{2}$ & Inspección ocular \\
\hline 6 & Biblioteca & Cataluña & 1992 & Administración & $500 \mathrm{~m}^{2}$ & $\begin{array}{l}\text { Estudio completo y } \\
\text { propuesta de refuerzo }\end{array}$ \\
\hline 7 & Viviendas & Andalucía & 1992 & Privada & $1.500 \mathrm{~m}^{2}$ & $\begin{array}{c}\text { Dictamen basado } \\
\text { exclusivamente en } \\
\text { ensayos }\end{array}$ \\
\hline 8 & Viviendas & Centro & 1992 & Privada & $5.500 \mathrm{~m}^{2}$ & $\begin{array}{l}\text { Identificación del } \\
\text { conglomerante y } \\
\text { estudio preliminar } \\
\text { de la degradación }\end{array}$ \\
\hline 9 & $\begin{array}{l}\text { Viviendas y } \\
\text { Oficinas }\end{array}$ & $\begin{array}{l}\text { Centro y } \\
\text { Cataluña }\end{array}$ & 1992 & Privada & 11 edificios & $\begin{array}{l}\text { Identificación del } \\
\text { conglomerante y } \\
\text { estudio preliminar } \\
\text { de la degradación }\end{array}$ \\
\hline 10 & Hotel & Andalucía & 1992 & Administración & $2.400 \mathrm{~m}^{2}$ & Inspección en curso \\
\hline 11 & Hospital & Centro & 1992 & Administración & $35.000 \mathrm{~m}^{2}$ & Inspección en curso \\
\hline 12 & Turístico & Cataluña & 1992 & $\begin{array}{l}\text { Organismo } \\
\text { Público }\end{array}$ & $400 \mathrm{~m}^{2}$ & Inspección en curso \\
\hline 13 & Viviendas & La Mancha & 1992 & Administración & $32.000 \mathrm{~m}^{2}$ & Inspección en curso \\
\hline
\end{tabular}




\section{INFORME DE LA PRIMERA FASE DEL ESTUDIO DEL ESTADO ACTUAL DE LOS FORJADOS DE 8 EDIFICIOS DE VIVIENDAS}

En septiembre de 1991 la Propiedad de un conjunto de edificios situados en Cataluña encargó a INTEMAC una inspección ocular preliminar que permitiera la redacción de una propuesta de plan de actuación para el estudio del estado actual de los forjados construidos con hormigón de cemento aluminoso y que presentaban síntomas de daños. Como consecuencia de la inspección INTEMAC propuso una primera fase del plan de actuación cuyo objeto erá el estudio del estado de conservación de viguetas y la propuesta de refuerzo de las mismas, realizado con un alcance limitado. En fases sucesivas del estudio, y si se considerase preciso, se llevarían a cabo las actividades necesarias para verificar aspectos relacionados con la capacidad portante de otros elementos de la estructura no contemplados en esta primera fase. $\mathrm{El}$ plan de actuación comprendía las actividades siguientes:

1.9) Inspección de la cara inferior de los vanos de forjado en las zonas en que presumiblemente se produce una degradación más intensa (zonas húmedas tales como cocinas, aseos, lavaderos, terrazas o balcones, techos de áticos bajo cubierta, zonas de paso de bajantes, cuartos de caldera o contadores, etc). Para ello debería procederse previamente a la eliminación de los revestimientos (enlucidos, enfoscados, falsos techos, etc) de la cara inferior de dichos vanos de forjado.

2.9) Inspección de la cara inferior de vanos de forjado en zonas secas con objeto de verificar el alcance de la degradación de las piezas resistentes de dichas zonas. Esta inspección correspondería a un muestreo reducido en el que se reconocería la cara inferior del forjado en tres puntos: apoyo dorsal, centro del vano y apoyo frontal, de tres vanos situados en zonas secas de cada vivienda. La inspección se llevaría a cabo a través de taladros de $20 \mathrm{~mm}$ de diámetro realizados en falsos techos en nueve puntos de cada vivienda de forma que pudiera practicarse la inspección ocular con endoscopio sin necesidad de producir grandes daños en falsos techos.
3.') Realización de ensayos químicos y de difracción de rayos $\mathrm{X}$ en ocho muestras del hormigón de viguetas correspondientes a cada uno de los edificios en estudio. Los análisis químicos tendrían por objeto la determinación de la naturaleza del conglomerante empleado como paso previo a la realización del difractograma de rayos $\mathrm{X}$ que permitiría establecer los tipos de aluminatos cálcicos presentes en la muestra.

4. ${ }^{\circ}$ Redacción de un informe de resultados, incluyendo el diagnóstico sobre el estado de conservación de las piezas inspeccionadas y recomendaciones sobre las actuaciones posteriores a seguir. El informe incluiría una propuesta de refuerzo tipo para las viguetas dañadas que precisen operaciones de consolidación inmediata, con croquis descriptivos de la solución prevista y recomendaciones sobre el procedimiento de ejecución.

Los edificios se concentran en dos manzanas y todos ellos presentan siete alturas sobre la rasante de la calle, con locales comerciales y portería en la planta baja y cuatro viviendas en cada una de las plantas restantes. La estructura de los edificios consisten, en general, en muros de fábrica de ladrillo macizo sobre los que apoyan forjados unidireccionales de viguetas autorresistentes prefabricadas de hormigón pretensado, con entrevigado formado por bóveda de una hoja de piezas cerámicas.

La práctica totalidad de las lesiones observadas en la inspección preliminar eran debidas a la degradación del hormigón de las viguetas y a la corrosión de las armaduras. Dado que la intensidad de los daños detectados en habitaciones "húmedas" se consideró crítica, se recomendó la adopción de medidas de apeo de seguridad con carácter urgente, así como proceder a una inspección del $100 \%$ de las viguetas en esas áreas. En el caso de habitaciones "secas" se propuso determinar mediante un muestreo reducido si existen en esas zonas piezas dañadas para estimar, al menos cualitativamente, la progresión del fenómeno de alteración del cemento aluminoso mediante las correspondientes determinaciones químicas y de difracción de rayos $\mathrm{X}$ sobre las muestras extraidas de las viguetas.

http://informesdelaconstruccion.revistas.csic.es 
Las conclusiones y recomendaciones de la primera fase del estudio se resumen en lo que sigue:

1) La causa fundamental de las lesiones existentes en viguetas de forjado es la degradación del cemento aluminoso utilizado como conglomerante del hormigón de dichas viguetas.

2) Los ensayos físico-químicos realizados indican la degradación de las viguetas muestreadas, tanto de las localizadas en zonas "húmedas" como de aquéllas dispuestas en zonas "secas".

3) A la vista de la manifiesta alteración del hormigón: aumento de porosidad, disminución de la basicidad, etc, y el consiguiente aumento de la susceptibilidad al ataque por corrosión de las armaduras, se recomendó el refuerzo de todas las viguetas dispuestas en zonas "húmedas" (aseos o cocinas) independientemente de que presentaran o no síntomas de degradación observables. Las viguetas localizadas en zonas "secas" se reforzarían solamente en el caso de que manifestasen daños. El informe recoge croquis en plantas con la localización de las zonas donde deben reforzarse las viguetas, así como croquis de definición de distintas tipologías de refuerzo dependiendo del material constituyente del elemento de apoyo y de la longitud de la vigueta a reforzar. La solución de refuerzo propuesta consiste en adosar inferiormente una vigueta metálica previo saneado del ala inferior de la vigueta de hormigón existente. La reparación de las zonas saneadas se llevaría a cabo con mortero de resina epoxi a fin de evitar problemas de compatibilidad del conglomerante aluminoso con otros tipos de cemento.

4) Es imprescindible acometer una campaña de inspecciones periódicas que permita detectar cualquier evolución desfavorable de los procesos de degradación señalados. Para ello deben dejarse registros practicables en todas las zonas que no se hayan reforzado entre las seleccionadas como de mayor nivel de riesgo. La frecuencia de dichas inspecciones deberá ser anual en zonas "secas" y semestral en zonas "húmedas" bajo cubierta, debiendo procederse a la inmediata reparación y refuerzo en caso de detectar un agravamiento de los daños.

La propuesta de refuerzo se basó, entre otras, en las conclusiones obtenidas tras la interpretación de los resultados de los análisis físico-químicos:
- Los análisis químicos revelan la utilización de cemento aluminoso como conglomerante en todas las muestras analizadas.

- Los valores de porosidad son sensiblemente superiores a los que corresponderían a hormigones de cemento aluminoso correctamente ejecutados y que no hubieran experimentado ningún proceso de degradación.

- Los difractogramas de rayos X indican la presencia en todas las muestras de algunos de los compuestos cristalinos característicos de la hidratación del cemento aluminoso. Igualmente ponen de manifiesto la presencia de compuestos calizos, cuarzo, tectosilicatos, micas y arcillas procedentes de los áridos empleados en la fabricación del hormigón. También aparecen compuestos cristalinos indicativos de que la pasta de cemento se encuentra en un avanzado estado de degradación, debido probablemente a la agresión química por hidrólisis alcalina. La ordenación de los compuestos cristalinos en el difractograma permite observar que 4 de las 10 muestras analizadas presentan un grado de alteración más avanzado que las restantes. Esta hipótesis se corrobora por los elevados valores de porosidad obtenidos en dichas muestras.

El proceso de degradación por hidrólisis alcalina (probablemente debido a la naturaleza de una fracción del árido empleado, a la difusión del anhídrico carbónico del aire por los poros accesibles del hormigón y/o a la posible disolución parcial de los álcalis presentes en el árido), provoca en el hormigón una disminución sensible de su resistencia mecánica con un incremento de la porosidad. Estos daños aumentan la susceptibilidad de las armaduras frente a la corrosión.

\section{ESTUDIO DE LA EXISTENCIA DE DAÑOS POR DEGRADACIÓN DE CEMENTO ALUMINOSO EN SEMIVIGUETAS DE LOS FORJADOS DE PLANTAS SEGUNDA A CUBIERTA DE UN EDIFICIO HOSPITALARIO}

En enero de 1992 el Instituto realizó una inspección preliminar del edificio citado a solicitud del contratista de las obras de remodelación de la residencia sanitaria. En el mes de febrero del mismo año el Instituto emitió el resultado de los ensayos efectuados sobre 25 muestras http://informesdelaconstruccion.revistas.csic.es 
de hormigón endurecido y durante febrero y marzo de 1992, a solicitud de la Dirección Facultativa, se llevaron a cabo trabajos encaminados a determinar por inspección visual la existencia de daños relacionados con la conversión del cemento aluminoso. Durante los meses de abril, mayo y junio se desarrollaron los restantes trabajos de campo previstos. Por expresa indicación del Peticionario, el ámbito del estudio se restringe a la identificación del tipo de conglomerante utilizado en viguetas y a la detección de eventuales procesos de degradación así como a su evaluación exclusivamente desde el punto de vista químico y no mecánico resistente, como inicialmente había propuesto el Instituto.

El inmueble en que se encuentran los forjados tiene nueve alturas sobre la rasante e incluye forjados de planta baja y primera resultantes de una etapa de construcción posterior en que se cubren los patios existentes, dando lugar en plantas baja y primera, a una planta del edificio de forma rectangular. La estructura está constituida generalmente por pórticos rectangulares de hormigón armado con vigas de canto y soportes de sección rectangular del mismo material. Los forjados están formados por semiviguetas de hormigón pretensado, prefabricadas, entrevigado de bovedillas de mortero y losa de hormigón in situ. El canto total del forjado es de $22 \mathrm{~cm}$, de los cuales $12,4 \mathrm{~cm}$ corresponden al canto de las semiviguetas que se disponen con intereje de $99 \mathrm{~cm}$.

La inspección ocular realizada en las distintas fases del estudio, permite establecer que la gran mayoría de las lesiones detectadas está asociada a la corrosión de los alambres de pretensado de las viguetas. La gradación de los daños observados varía desde las manchas de óxido en las suelas hasta la fisuración y posterior desprendimiento del hormigón de recubrimiento, detectándose en muchos casos una pérdida significativa de la sección resistente de los alambres y, en alguna ocasión, su rotura total. Se inspeccionaron en total 439 viguetas obteniéndose el siguiente resultado estadístico:

Viguetas sin defectos aparentes: Viguetas con machas de óxido:

Viguetas con daños graves:

Los análisis físico-químicos realizados permiten establecer lo siguiente:

1- Se detecta la presencia de cemento aluminoso en todas ellas.
2- Todas las muestras presentan síntomas de haber sufrido procesos de carbonatación en gran extensión, con una única excepción.

3- Cuatro de las muestras presentan indicios de alteración por conversión cristalina.

4- Los valores de porosidad obtenidos son sensiblemente elevados, en relación con los valores normales de hormigón de cemento aluminoso no transformado, e indicativos de la existencia de procesos de degradación.

La degradación del cemento aluminoso es consecuencia de tres diferentes tipos de transformaciones químicas:

La conversión cristalina del cemento aluminoso, por la que los carboaluminatos originalmente cristalizados en forma metaestable (sistema hexagonal) se transforman a fases estables (sistema cúbico). En estas transformaciones se libera hidróxido de aluminio y agua y se origina una disminución importante del sólido cementante, del orden del $40 \%$, provocando una importante elevación de la porosidad, una disminución notable de las resistencias mecánicas y una pérdida sensible de la capacidad de protección de las armaduras frente a la corrosión.

La carbonatación del cemento aluminoso, que en medio húmedo comporta la destrucción de los productos resultantes de la transformación de las fases hexagonales en cúbicas y da lugar fundamentalmente a aragonito, calcita y a una matriz de alúmina anhidra. Si en un hormigón de cemento portland la carbonatación trae consigo la pérdida de basicidad y de protección frente a la corrosión, en un hormigón de cemento aluminoso el fenómeno comporta mayor daño como consecuencia de la inferior basicidad del conglomerado sin degradar y de la pérdida del poder cementante de la propia pasta.

La tercera de las transformaciones que degrada el hormigón es la hidrólisis alcalina de los aluminatos del calcio hidratado y la generación de los carbonatos alcalinos en contacto con el anhídrido carbónico presente en la atmósfera. Estos procesos conducen a una pérdida de tenacidad y cohesión del hormigón y a una pérdida de resistencia.

Adicionalmente se realizaron análisis de muestras correspondientes a pilares y vigas, obteniendo la conclusión de que fueron fabricadas con cemento portland. 
Ante los aspectos observados y tras el análisis de los resultados se establecieron las siguientes conclusiones y recomendaciones:

1.) La causa fundamental de las lesiones existentes en viguetas de forjados es la oxidación de las armaduras embebidas en hormigón fabricado con cemento aluminoso, fenómeno favorecido por los procesos de degradación de dicho material.

2.) Los ensayos físico-químicos indican la degradación química del hormigón fabricado con cemento aluminoso existente en las viguetas muestreadas, tanto las localizadas en zonas "húmedas" como en aquellas dispuestas en zonas "secas".

3.) Se recomienda el refuerzo de todas las viguetas dispuestas en zonas "húmedas" existan o no en la actualidad síntomas de degradación detectables por simple inspección ocular. Las viguetas localizadas en zonas "secas" con alguna manifestación del proceso deberían ser igualmente reforzadas.

Se recomienda realizar un refuerzo generalizado de las viguetas del inmueble o bien, opcionalmente, acometer una campaña de ensayos mecánicos que tenga por objeto estimar la capacidad resistente residual de las viguetas dañadas. La campaña de ensayos recomendada permitiría establecer un criterio más preciso y fundado sobre la localización de las viguetas a reforzar.

En cualquier caso al no poder garantizarse la vida útil de las viguetas del inmueble deberá acometerse una campaña de inspecciones periódicas, dejando registros practicables en todas las zonas que no se hayan reforzado. Se mantiene el criterio sobre la frecuencia de dichas inspecciones: anual en zonas "secas" y semestral en zonas "húmedas" (bajo cubierta).

\section{ESTUDIO DE LA EXISTENCIA DE DAÑOS POR DEGRADACIÓN DE CEMENTO ALUMINOSO EN VIGUETAS DE FORJADO}

En octubre de 1991 INTEMAC realizó una inspección preliminar de los daños que presentaban diversos (c) Consejo Superior de Investigaciones Científicas

Licencia Creative Commons 3.0 España (by-nc) forjados de 18 edificios de la ciudad. Como resultado de la inspección el Instituto elaboró un plan de actuación para el estudio de la existencia de daños por degradación de cemento aluminoso en viguetas. Los trabajos de obra y ensayos de laboratorio previstos se realizaron durante el mes de diciembre de 1991 y se prolongaron a lo largo de los meses de enero, febrero y marzo de 1992. El alcance de las inspecciones y tomas de muestras tuvo que ser limitado por falta de autorización para el acceso a la vivienda en algunos casos.

Los trabajos efectuados consisten en la inspección ocular de la cara inferior de las viguetas de los 17 inmuebles, en la identificación del conglomerante utilizado y en la detección de eventuales procesos de degradación en aquellos elementos en que se hubiera comprobado la presencia de cemento aluminoso.

La inspección y toma de muestras se intensificó en las zonas "húmedas" de los edificios situadas en cubierta, en una planta intermedia y en la planta semisótano. En cada edificio se elegía una muestra para su ensayo en laboratorio, previo apeo de la vigueta elegida y con posterior restitución de la zona dañada por el procedimiento previamente autorizado por INTEMAC. Sobre las muestras obtenidas se realizarían los siguientes ensayos:

- Análisis químico para la determinación del conglomerante utilizado.

- Análisis por difracción de rayos X.

- Análisis termo-gravimétrico.

- Determinaciones de la resistencia a compresión sobre dos probetas talladas de cada muestra.

- Determinaciones de porosidad y densidad.

- Determinaciones de las características mecánicas residuales de los alambres de pretensado.

- Determinaciones geométricas de la sección y esquemas de armado de las viguetas.

Los resultados obtenidos sobre las 18 muestras finalmente analizadas llevan a la conclusión de que en 6 de ellas el conglomerante utilizado es tipo portland y en las 12 restantes es aluminoso. De estas 12, en 7 se presentan indicios de degradaciones puntuales por hidrólisis alcalina (únicamente en zonas en contacto con cemento portland), en 3 se detectan daños por conversión cristalina, y en una muestra se observan fenómenos de carbonatación.

http://informesdelaconstruccion.revistas.csic.es 
No obstante hay que resaltar que las alteraciones citadas no son excluyentes entre sí. Por último, en una de las muestras de hormigón de cemento aluminoso no pudieron obtenerse datos como consecuencia del tamaño de la misma.

En los ensayos mecánicos realizados se obtienen valores de la resistencia sensiblemente inferiores en viguetas con cemento aluminoso que en viguetas con cemento portland. También la dispersión de resistencias es muy elevada, oscilando entre $243 \mathrm{kp} / \mathrm{cm}^{2}$ y $366 \mathrm{kp} / \mathrm{cm}^{2}$ en hormigones de cemento aluminoso y siendo de 496 $\mathrm{kp} / \mathrm{cm}^{2}$ en hormigones de cemento portland. El límite elástico de los alambres que constituyen las armaduras oscila entre $11.800 \mathrm{~kg} / \mathrm{cm}^{2}$ y $18.300 \mathrm{~kg} / \mathrm{cm}^{2}$. En las determinaciones efectuadas las viguetas con sección transversal en simple $T$ fueron fabricadas con cemento portland, mientras que las de sección transversal en doble T fueron fabricadas empleando como conglomerante cemento aluminoso. En un caso aislado se detecta una vigueta doble $\mathrm{T}$ fabricada con cemento portland pero en este caso se trata de una vigueta simplemente armada y no pretensada.

La distribución de las muestras de hormigones de cemento portland y hormigones de cemento aluminoso es totalmente aleatoria, habiéndose detectado en 12 de los inmuebles procesos de degradación de hormigones con cemento aluminoso. El Instituto recomienda que se analice el nivel de seguridad, las condiciones de durabilidad y la necesidad, en su caso, de llevar a cabo actuaciones de reparación o refuerzo en los forjados de los edificios de referencia. Estos aspectos quedan fuera del alcance del estudio realizado, cuya conclusión es de que en los forjados se han empleado viguetas de hormigón de cemento aluminoso y en estos casos se detectan procesos de degradación del material.

\section{CONCLUSIÓN GENERAL}

Como conclusión de lo expuesto hasta ahora resulta que es necesario en todos los estudios sobre daños producidos en hormigón de cemento aluminoso llevar a cabo una inspección visual detallada de los elementos afectados. La inspección debe ser realizada por personal de suficiente formación básica y con experiencia en la investigación de casos de patología atribuible al empleo de cemento aluminoso. La inspección visual permitirá clasificar los daños y establecer un proceso racional de toma de muestras y ensayos de laboratorio, cuyo principal aspecto es establecer si realmente en la obra se utilizó como conglomerante el cemento aluminoso. Aspectos secundarios son el estudio del nivel de degradación del hormigón de cemento aluminoso y el establecimiento del nivel de daños por corrosión existente en las armaduras. Los estudios anteriores deben complementarse con un análisis de la seguridad en el estado real de la estructura que permita establecer la urgencia y procedimientos más adecuados de reparación o refuerzo.

La simple inspección visual puede permitir, en base a la tipología de los elementos afectados, una primera estimación de si se ha empleado o no cemento aluminoso durante la construcción. Las reglas antiguamente aplicadas y recogidas por la bibliografía sobre la estimación mediante inspección ocular del tipo de conglomerante empleado en el hormigón no han resultado eficaces en los casos investigados.

\section{BIBLIOGRAFÍA}

(1) COLLINS, R.J. y WUTT

Update on assessment of high alumina cement concrete. BRE Information Paper. Latest Research Information and how to apply it. IP8/88. Julio 1988. Building Research Stablishment (BRE). Department of the Environment.

(2) BATE, S.C.C.

High alumina cement concrete in existing building superestructures.

BRE Report. London, HMSO, 1984.
(3) Aluminosis.

Almacén de Materiales para la Construcción. No 24. Noviembre 1992. Ediciones Rodasa, Barcelona.

(4) S.A. de Hormigones Especiales (SAHE) Viguetas y postes de hormigón pretensado. Publicación 4-C. SACE, María de Molina 26, Madrid.

(5) PRATT, P.L.

High alumina cement concrete in construction. Structural Survey. pp. 261 a 267, 1991. 
(6) LAFARGE SPECIAL CEMENTS

Ciment fondu Lafarge. Guide for use. Construction applications.

CA4 890. CA1 790. Lafarge Special Cements. London, 1990.

(7) TALERO, R., TRIVIÑO, F., PALACIOS, J., DÍAZ GARCÍA, F. La "aluminosis" del cemento aluminoso o un término nuevo para una clásica enfermedad.

Materiales de Construcción, Vol. 39, No 216, Octubre - Noviembre - Diciembre 1989. Instituto Eduardo Torroja. Madrid.

(8) LEA, F.M.

The chemistry of cements and concrete.

Edward Arnold Ed. Londres 1963.

(9) KEIL, Fritz

Zement

Editores Técnicos Asociados (ETA). Barcelona 1973.
(10) NEVILLE, Adam

High Alumina Cement Concrete

The Construction Press. Lancaster (U.K.), 1975.

(11) ROBERT, M.H.

Método químico rápido para la detección de cemento aluminoso en el hormigón.

Traducción. Boletín ICCE. Murcia, 1991.

(12) AFNOR

Empleo del Cemento Aluminoso Fundido

Traducción J.M. Fernández París, INTEMAC, de la norma francesa P15-316 (Abril 1991).

(13) AFNOR

Ciment alumineux fondu.

Norma francesa NF, P15-315 (Abril 1991).

\section{publicaciones del ICCET/CSIC}

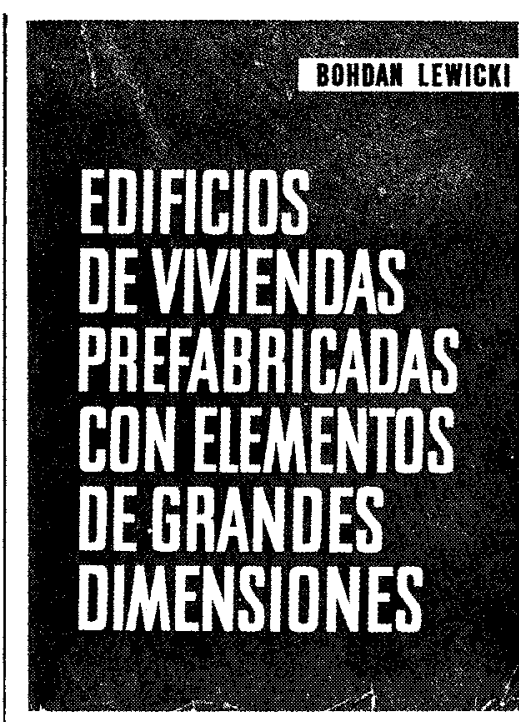

Bohdan Lewicki

Este libro trata de los problemas relativos a la construcción de los edíficios de viviendas o públicos realizados con elementos prefabricados de grandes dimensiones. Se han estudiado los problemas de arriostramiento, así como los que plantea la resistencia de los elementos y de la estructura; se han examinado las cuestiones de orden higroter. mico, acústico y de resistencia al fuego; también se ha profundizado en el estudio de la estanquidad de los muros exteriores y de las juntas.

La obra incluye numerosas ilustraciones que dan detalles de diversas soluciones, así como ejemplos de cálculo, tablas de valores numéricos, diagramas y ábacos.

Un volumen encuadernado en tela, de

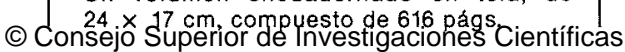
Licencia Creative Commons 3.0 España (by-nc)
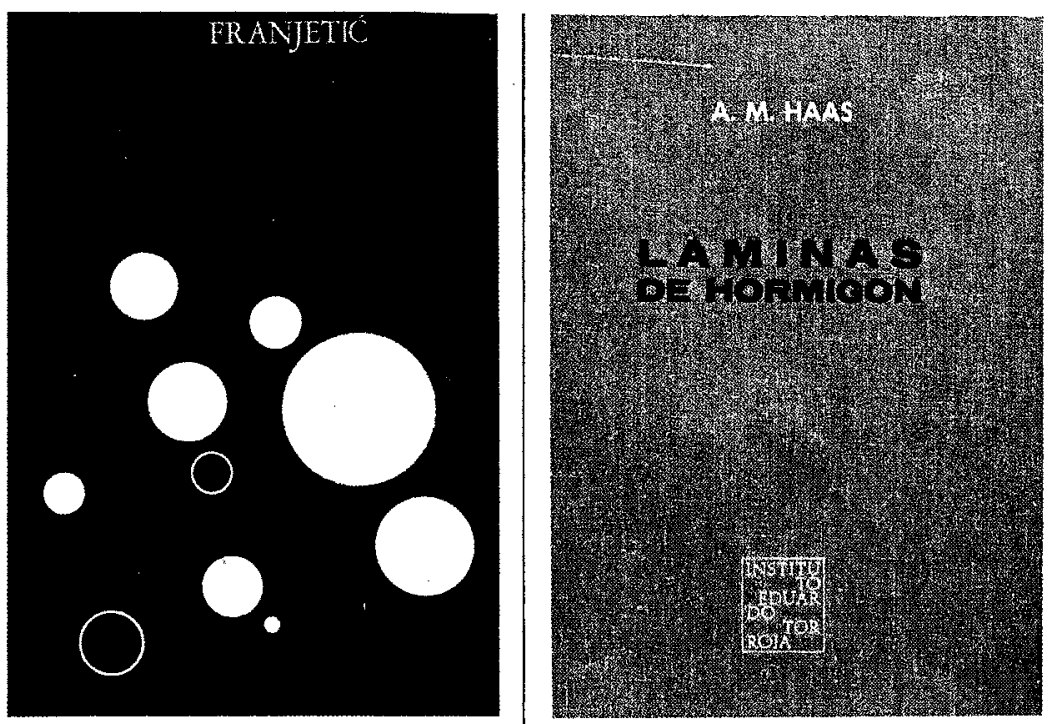

Zorislav Franjetić

En la obra de Franjetić se expone de una forma minuciosa, ordenada y sistemática, todo un cuerpo de doctrina que reúne el conocimiento actual sobre el endurecimiento rápido del hormigón. Parte el autor de los principios básicos y llega a las últimas consecuencias y realidades técnicas y económicas.

Es una obra de consulta, tanto para el investigador sobre la materia, como para el proyectista y el realizador y montador de plantas e instalaciones y equipos de curado y endurecimiento rápido.

Un volumen sncuadernado en cartóné, de $17 \times 24,5 \mathrm{~cm}$, compuesto de 385 págs. 110 figuras y 10 tablas.

\section{A. M. Haas}

Al escribir este libro el autor intentó poner a disposición de los estudiantes y de los ingenieros unos conocimientos prácticos, adecuados para servir de guia en el diseño y construcción de láminas delgadas de hor. migón.

El autor está convencido de que el éxito en el diseño de una lámina exige, por parte del proyectista, un examen de las tres fases por las que pasa la materialización de la lámina: el diseño, el análisis estructural y la construcción de la estructura.

Un volumen encuadernado en tela, de $17 \times 24,5 \mathrm{~cm}$, compuesto de 420 págs., 141 figuras, 22 fotografias y 6 tablas. 